\title{
Proyecto SAFO: La diversidad sexual a lo largo del ciclo vital en España
}

\author{
Juan Enrique Nebot-Garcia \\ Grupo Salusex. Departamento de Psicología Básica, Clínica y Psicobiología, Universitat \\ Jaume I, Castellón, España. \\ junebot@uji.es \\ Marcel Elipe-Miravet \\ Grupo Salusex. Departamento de Psicología Básica, Clínica y Psicobiología, Universitat \\ Jaume I, Castellón, España. \\ elipe@uji.es \\ Naiara Martínez-Gómez \\ Grupo Salusex. Departamento de Psicología Básica, Clínica y Psicobiología, Universitat \\ Jaume I, Castellón, España. \\ gomezn@uji.es \\ Rafael Ballester-Arnal \\ Grupo Salusex. Departamento de Psicología Básica, Clínica y Psicobiología, Universitat \\ Jaume I, Castellón, España. \\ rballest@uji.es
}

\section{RESUMEN}

La orientación sexual ha sido, sobre todo en épocas pasadas, una dimensión de la sexualidad muy vigilada y restringida, castigándose cualquier comportamiento o deseo que se alejara de la heterosexualidad. Sin embargo, en la actualidad, las personas jóvenes disponen de mucha más información y apertura hacia la diversidad sexual. Por ello, este trabajo pretende evaluar la diversidad sexual existente en España en función del ciclo vital. Un total de 6274 personas, con una edad media de 37.85 años $(D T=$ 13.03), contestaron una encuesta ad hoc sobre diversidad sexual y experiencias asociadas, dentro del Proyecto SAFO (Salusex, UJI-UV). Para analizar los datos, se realizaron seis grupos de edad (18-25, 26-35, 36-45, 46-55, 56-65, más de 65 años) con $50 \%$ de hombres cis y $50 \%$ de mujeres cis en cada grupo. En función de la edad, se observa que las personas mayores son quienes más se identifican con la heterosexualidad y los más jóvenes los que se identifican más con etiquetas menos normativas y tradicionales. Estas diferencias también se observan al evaluar la atracción sexual en el continuo de Kinsey, siendo los grupos de mayor edad los que muestran mayor porcentaje de atracción exclusiva hacia el otro sexo. Además, los grupos más jóvenes son los que más dudas y más cambios han experimentado con la orientación sexual. La población joven parece reflexionar y cuestionarse más su orientación sexual y muestra mayor diversidad sexual que la población más adulta. Este efecto puede ser debido a la mayor represión y a la escasez de información sobre diversidad sexual existente en épocas pasadas, lo que limitaba las experiencias y la libertad de los más mayores durante su adolescencia y juventud, una época clave para el desarrollo de la identidad y la formación de la orientación sexual.

Palabras clave: orientación sexual; fluidez sexual; edad; dudas; cambios

\section{ABSTRACT}

Sexual orientation has been, especially in the past, a highly guarded and restricted dimension of sexuality, with any behavior or desire that departed from heterosexuality 
being punished. However, nowadays, young people have much more information and openness towards sexual diversity. For this reason, this study aims to evaluate sexual diversity in Spain according to life cycle. A total of 6274 people, with a mean age of 37.85 years $(\mathrm{SD}=13.03)$, answered an ad hoc survey on sexual diversity and associated experiences, within the SAFO Project (Salusex, UJI-UV). To analyze the data, six age groups were performed (18-25, 26-35, 36-45, 46-55, 56-65, over 65 years) with $50 \%$ cis men and $50 \%$ cis women in each group. Depending on age, it is observed that older people identify more with heterosexuality and younger people identify more with less normative and traditional labels. These differences are also observed when evaluating sexual attraction on the Kinsey continuum, with older groups showing the highest percentage of exclusive attraction to the other sex. In addition, the youngest groups are the ones who have experienced the most doubt and the most change with sexual orientation. The younger population seems to reflect and question their sexual orientation more and shows more sexual diversity than the older population. This effect may be due to the greater repression and scarcity of information on sexual diversity in the past, which limited the experiences and freedom of older people during their adolescence and youth, a key time for the development of identity and the formation of sexual orientation.

Key words: sexual orientation; sexual fluidity; age; doubts; changes

\section{INTRODUCCIÓN}

Durante las primeras investigaciones sobre la orientación sexual, esta se había concebido como una dimensión estructurada, estanca, y situada en el marco de la heteronormatividad, castigando cualquier deseo que se alejara de la heterosexualidad. Se entendía la orientación sexual como algo inherente al sexo biológico, estable en el tiempo y limitado a categorías binarias cerradas, estableciendo dos etiquetas dicotómicas y excluyentes: homosexual vs heterosexual (Carroll, 2015). Unos de los primeros autores en cuestionar y defender las diversidades dentro de la sexualidad fueron Kinsey et al. (1948; 1953) y Klein (1978), que ayudaron a romper la clasificación binaria de la orientación establecida anteriormente, cuestionándose así los significados de las etiquetas homosexual, bisexual y heterosexual, y otorgándoles flexibilidad. Así pues, confirmaron que estas etiquetas estaban marcadas por las vivencias y se empezó a concebir la orientación sexual como una dimensión dentro de un continuo, con diferentes posiciones intermedias. Esto hecho hizo que se empezaran a tener en cuenta otras dimensiones como son la atracción sexual, las conductas sexuales y las fantasías, pudiendo éstas fluctuar en una persona a lo largo de los años. En línea con lo defendido por estos autores, varias investigaciones sostienen que las personas que se identifican con una misma etiqueta de orientación sexual, pueden tener diferencias en cuanto a conductas sexuales, fantasías, atracciones sexuales y románticas (Ballester-Arnal y Gil-Llario, 2016; Nebot-Garcia et al., 2018).

A pesar de la evolución en los estudios sobre diversidad sexual y la premisa de la flexibilidad de la orientación sexual, a día de hoy aún seguimos bajo el marco de la heteronormatividad (Herz y Johansson, 2015). Aquellas personas que sienten que pueden salirse de este marco heterosexual, pasan por un cuestionamiento de su sexualidad antes de definirse (Morgan et al., 2010). Durante este proceso, quienes finalmente se encuentran fuera de la heteronormatividad sufren el estigma asociado a las minorías sexuales y de género, siendo este estigma una herencia que sigue a día de hoy 
teniendo un papel relevante en el desarrollo de la sexualidad de las personas (Bauermeister, 2010). Uno de los factores que resultan de esta discriminación es el desarrollo de la homofobia interiorizada, siendo ésta la interiorización de los estereotipos y prejuicios que emite la sociedad sobre las personas LGTBI (lesbianas, gays, trans, bisexuales e intersexuales) (Walch et al., 2016). Este rechazo conlleva que muchas personas no se permitan experimentar su sexualidad o en ocasiones tengan que marcharse a entornos más tolerantes para poder vivir acorde a su identidad (sexilio).

Además, todas esas situaciones discriminatorias tienen un impacto en las personas pertenecientes a una minoría sexual y de género, pudiendo verse afectada su salud mental. Por ejemplo, varios estudios han observado en este colectivo falta de autoestima (Martxueta y Etxeberria, 2014), pobre autoimagen (Frederick y Essayli, 2016), mayor sintomatología depresiva (Carroll, 2015; Espada et al., 2012; Marchueta, 2014; Martxueta y Etxeberria, 2014), mayor ansiedad (Marchueta, 2014; Martxueta y Etxeberria, 2014), abuso de sustancias (Carroll, 2015), e incluso conductas suicidas (Carroll, 2015; Reisner et al., 2014).

Aunque se han realizado estudios parciales sobre algunas de estas cuestiones en el contexto español, muchos sólo analizan la orientación sexual y la identidad de género desde una perspectiva binaria y categorial, sin incluir alternativas como la pansexualidad, la asexualidad, las personas transgénero o la intersexualidad. Por otro lado, no son frecuentes los estudios comparativos en los que se analice la problemática asociada a las minorías sexuales frente a las personas que se adscriben a la heterosexualidad normativa. Tampoco es habitual encontrar estudios en los que se indague tanto en los problemas de salud mental como en las variables asociadas a una mayor resiliencia frente al estrés soportado por las minorías sexuales, desde una perspectiva de la psicología positiva. Finalmente resulta necesario un estudio a nivel nacional con una muestra muy amplia que sirva como referente a investigadores, educadores, clínicos y políticos, con el fin de implementar medidas legislativas y educativas conducentes a prevenir el estigma a que es sometida la población LGTBI, proteger derechos humanos básicos asociados a la libertad sexual de estos individuos e intervenir ante los problemas de salud mental asociados a las minorías sexuales y de género.

Dada la importancia de estudiar la diversidad sexual y de género en el contexto español, el equipo de investigación Salusex (Universitat Jaume I y Universitat de València) crea el Proyecto SAFO, liderado en la UJI por el profesor Rafael Ballester y en la U. de Valencia por la profesora $\mathrm{M}^{\mathrm{a}}$ Dolores Gil, cuyo objetivo es analizar de forma amplia y comprehensiva todas las realidades que se alejan de la heteronormatividad, así como la existencia de posibles problemas de salud mental asociados a las minorías sexuales y de género como consecuencia de la discriminación y el estigma existente en la sociedad hacia el colectivo LGTBI.

En este trabajo se darán además unos resultados preliminares sobre la diversidad sexual y las vivencias asociadas en función de la etapa vital, cogiendo como referencia la orientación sexual con la que se identifican las personas, la atracción sexual según Kinsey, y las dudas con la orientación y los cambios experimentados en la misma.

\section{PROCEDIMIENTO}

\section{MÉTODO}


Para la recogida de la muestra, se utilizaron dos estrategias. Por una parte, se difundieron dos anuncios a través de Facebook e Instagram en el que se solicitaba la participación en un estudio sobre sexualidad. Por otra parte, para conseguir mayor muestra de personas con diversidad sexual, se contactó con asociaciones y organizaciones LGTBI de todo el estado español para que difundieran la investigación en sus redes sociales y entre sus contactos.

En ambos casos, una vez clicaban en el anuncio, se les informaba del carácter anónimo, voluntario y confidencial de la investigación y se les pedía el consentimiento informado. A continuación, accedían al cuestionario on-line desde sus dispositivos. Esta investigación contaba con la aprobación de la Comisión Deontológica de la Universitat Jaume I (Castellón, España) y, en todo momento, se siguieron los principios éticos de la Declaración de Helsinki y se tomaron las medidas oportunas que marca la legislación más reciente para cumplir con la protección de datos.

A través de un muestreo aleatorio simple, se recogieron un total de 12500 respuestas, 8948 mediante los anuncios y 3552 a través de las asociaciones LGTBI. Para el presente estudio, en el que se pretende dar unos resultados preliminares de la diversidad sexual existente en la población española, solo se tuvieron en cuenta las respuestas obtenidas mediante los anuncios y a la población con una identidad cis para sesgar lo menos posible los resultados de diversidad. Pese a haber recogido un total de 8948 respuestas, solo 7835 cumplían con los criterios de inclusión: identificarse como hombre cis o mujer cis, ser mayor de edad y residir en España. Posteriormente, se realizaron seis grupos de edad (18-25, 26-35, 36-45, 46-55, 56-65, más de 65 años) y se seleccionó la muestra para que hubiera en cada grupo un 50\% de hombres cis y un $50 \%$ de mujeres cis, quedando finalmente un total de 6274 personas.

\section{PARTICIPANTES}

Del total de la muestra obtenida, se seleccionaron un total de 6274 españoles de entre 18 y 85 años $(M=37.85 ; D T=13.03)$, siendo el $50 \%$ hombres cis y el $50 \%$ mujeres cis. La mayoría de participantes tenían estudios universitarios, eran ateos o agnósticos, con una ideología política de izquierdas y vivían en una localidad urbana de interior.

\section{INSTRUMENTOS DE EVALUACIÓN}

En el proyecto SAFO, todos los participantes cumplimentaron un cuestionario ad hoc sobre diversidad sexual y experiencias asociadas, que constaba de 13 áreas temáticas:

Historia sexual y de pareja. Se preguntó sobre la edad de inicio sexual, las características y el contexto en el que se produjeron y su historia de pareja.

Diversidad sexual y de género. En esta dimensión se abordaron diferentes variables sobre diversidad sexual, como la identidad y expresión de género, la orientación sexual, así como otras variables asociadas.

Uso de aplicaciones de citas. Se evaluaron el tipo de aplicaciones de citas que se usaban y las conductas sexuales que se derivaban de ellas.

Imagen corporal y sexual. Mediante dos preguntas se evaluó la satisfacción corporal y con los genitales. 
Vivencias de la orientación sexual y la identidad de género. A las personas LGTBI, se les preguntó sobre el proceso de toma de conciencia de su orientación o identidad y de la salida del armario.

Reacción familiar ante la salida del armario. En el caso de haber salido del armario, se les pasaba la adaptación de Salusex (Nebot-Garcia et al., 2021a) al cuestionario Perceived Parental Reactions Scale (Willoughby et al., 2006).

LGTBIfobia. Se evaluó la LGTBIfobia manifiesta y sutil hacia diferentes grupos del colectivo LGTBI.

Bullying o acoso. Entre aquellas personas que habían sufrido bullying o acoso, se evaluaron las características de ese acoso.

Utilización de los servicios médicos. Se evaluó en la población LGTBI si, debido a su condición sexual, habían sufrido discriminación por el personal médico.

Estrés asociado a las minorías sexuales. Para evaluar el estrés asociado a las minorías sexuales se utilizó la adaptación realizada por Salusex (Nebot-Garcia et al., 2021b) al cuestionario The LGBT Minority Stress Measure (Outland, 2016).

Síntomas psicopatológicos. A través del Symptom Assessment-45 Questionnaire (SA-45, Davison et al., 1997; adaptado por Sandín et al., 2008) se evaluaron diferentes síntomas psicopatológicos en toda la población.

Ideación e intentos suicidas. A toda la población se les evaluaron la conducta suicida.

Bienestar emocional. A través de la versión española de la Escala de Bienestar de Ryff (Ryff, 1989; adaptado por Díaz et al., 2006) se evaluó el bienestar emocional.

Sin embargo, para el presente trabajo solamente se utilizaron cuatro ítems:

Orientación sexual categórica. A través de una pregunta de elección única se les preguntaba la orientación sexual con la que más se identificaban, debiendo elegir entre "heterosexual", "homosexual", "bisexual", "pansexual", "asexual" y "otra".

Atracción sexual según Kinsey. Mediante una escala continua de siete puntos se les pedía que indicaran hacia quién sentían atracción sexual, pudiendo elegir entre "siento atracción solo hacia el otro sexo", "siento atracción casi siempre hacia el otro sexo y en muy contadas ocasiones hacia mi mismo sexo", "siento atracción algo más hacia el otro sexo que hacia mi mismo sexo", "siento atracción hacia cualquier sexo", "siento atracción algo más hacia mi mismo sexo que hacia el otro sexo", "siento atracción casi siempre hacia mi mismo sexo y en muy contadas ocasiones hacia el otro mismo sexo" y "siento atracción solo hacia mi mismo sexo". Además, fuera de este continuo existían dos opciones más: "no siento atracción por ningún sexo" y "otra".

Dudas con la propia orientación sexual. Con una escala Likert de cuatro puntos se evaluaba si, en algún momento de sus vidas, habían tenido dudas sobre su orientación sexual. Las opciones de respuesta eran "nada", "algo", "bastante" y "mucho". 
Cambios en la propia orientación sexual. Por último, a través de una pregunta dicotómica, se les preguntaba si en algún momento de sus vidas habían experimentado cambios en su orientación sexual, teniendo que contestar con "sí" o "no".

\section{ANÁLISIS ESTADÍSTICO}

En primer lugar, se utilizó el programa estadístico SPSS versión 27 para realizar análisis descriptivos de las principales variables sociodemográficas. A continuación, se realizaron tablas de contingencias para conocer los porcentajes en la orientación sexual categórica, el continuo de atracción sexual, las dudas con la orientación sexual y los cambios en la orientación sexual. Por último, para analizar las diferencias en función de los grupos etarios, se realizaron pruebas de Chi cuadrado en cada una de las variables anteriormente mencionadas.

\section{RESULTADOS}

En términos generales, más de la mitad de la muestra (57.8\%) se identifica como heterosexual. De hecho, a excepción del grupo de 18 a 25 años, la heterosexualidad es la orientación sexual más prevalente en todos los grupos etarios (véase Tabla 2). En cambio, en el grupo más joven, de 18 a 25 años, la orientación con la que más gente se identifica es con la bisexualidad. Además, se observa una tendencia casi general en la que existe más gente que se identifica con la heterosexualidad a medida que incrementa la edad de los grupos, siendo estas diferencias estadísticamente significativas $\left(\chi^{2}=\right.$ $1136.58 ; 25 \mathrm{gl} ; p<.001)$.

Tabla 2. Categorías de orientación sexual en función de los grupos de edad.

\begin{tabular}{lccccccccccccc}
\hline & \multicolumn{3}{c}{$18-25$} & $26-35$ & \multicolumn{2}{c}{$36-45$} & $46-55$ & $56-65$ & \multicolumn{2}{c}{ Más de 65} \\
\cline { 2 - 14 } & $n$ & $\%$ & $n$ & $\%$ & $n$ & $\%$ & $n$ & $\%$ & $n$ & $\%$ & $n$ & $\%$ \\
\hline Heterosexual & 397 & 24.9 & 647 & 62.3 & 1384 & 67.7 & 772 & 77.2 & 357 & 70 & 69 & 76.7 \\
Homosexual & 296 & 18.6 & 134 & 12.9 & 318 & 15.6 & 81 & 8.1 & 51 & 10 & 8 & 8.9 \\
Bisexual & 720 & 45.2 & 190 & 18.3 & 253 & 12.4 & 117 & 11.7 & 84 & 16.5 & 10 & 11.1 \\
Pansexual & 95 & 6 & 37 & 3.6 & 47 & 2.3 & 12 & 1.2 & 6 & 1.2 & 0 & 0 \\
Asexual & 40 & 2.5 & 11 & 1.1 & 19 & 0.9 & 8 & 0.8 & 3 & 0.6 & 0 & 0 \\
Otra & 44 & 2.8 & 19 & 1.8 & 23 & 1.1 & 10 & 1 & 9 & 1.8 & 3 & 3.3 \\
\hline
\end{tabular}

Si se tiene en cuenta la atracción sexual en función del continuo de Kinsey, se observa que la atracción exclusiva hacia el otro sexo es la opción más prevalente, tanto en la muestra general (43\%), como en los distintos grupos etarios (véase Tabla 3). Del mismo modo que ocurría con la heterosexualidad, a medida que incrementa la edad de los grupos, hay una tendencia casi general en la que más gente manifiesta una atracción exclusiva hacia el otro sexo, siendo estas diferencias estadísticamente significativas $\left(\chi^{2}\right.$ $=1004.83 ; 40 \mathrm{gl} ; p<.001)$.

Tabla 3. Atracción sexual según Kinsey en función de los grupos de edad.

\begin{tabular}{lcccccccccccc}
\hline & \multicolumn{3}{c}{$18-25$} & \multicolumn{2}{c}{$26-35$} & \multicolumn{2}{c}{$36-45$} & \multicolumn{4}{c}{$46-55$} & \multicolumn{2}{c}{$56-65$} & \multicolumn{2}{c}{ Más de 65 } \\
\cline { 2 - 13 } & $n$ & $\%$ & $n$ & $\%$ & $n$ & $\%$ & $n$ & $\%$ & $n$ & $\%$ & $n$ & $\%$ \\
\hline Solo hacia el otro sexo & 303 & 19 & 440 & 42.4 & 1008 & 49.3 & 603 & 60.3 & 280 & 54.9 & 66 & 73.3 \\
Casi siempre hacia el otro sexo & 294 & 18.5 & 271 & 26.1 & 491 & 24 & 208 & 20.8 & 107 & 21 & 11 & 12.2 \\
Algo más hacia el otro sexo & 243 & 15.3 & 63 & 6.1 & 76 & 3.7 & 35 & 3.5 & 21 & 4.1 & 1 & 1.1 \\
Hacia cualquier sexo & 188 & 11.8 & 72 & 6.9 & 76 & 3.7 & 25 & 2.5 & 17 & 3.3 & 2 & 2.2 \\
Algo más hacia mi mismo sexo & 153 & 9.6 & 30 & 2.9 & 51 & 2.5 & 29 & 2.9 & 27 & 5.3 & 0 & 0
\end{tabular}


Casi siempre hacia mi mismo sexo

\begin{tabular}{cccccccccccc}
214 & 13.4 & 74 & 7.1 & 119 & 5.8 & 32 & 3.2 & 25 & 4.9 & 3 & 3.3 \\
154 & 9.7 & 62 & 6 & 197 & 9.6 & 57 & 5.7 & 24 & 4.7 & 6 & 6.7 \\
16 & 1 & 6 & 0.6 & 6 & 0.3 & 5 & 0.5 & 4 & 0.8 & 0 & 0 \\
27 & 1.7 & 20 & 1.9 & 20 & 1 & 6 & 0.6 & 5 & 1 & 1 & 1.1 \\
\hline
\end{tabular}

Por otra parte, un $40.1 \%$ de la muestra manifiesta haber tenido, en mayor o menor medida, dudas con su orientación sexual (véase Tabla 4). En este caso, el grupo más joven es el que más dudas ha experimentado, observándose una disminución a medida que aumenta la edad de los grupos, siendo las diferencias estadísticamente significativas $\left(\chi^{2}=1354.01 ; 15 \mathrm{gl} ; p<.001\right)$.

Tabla 4. Dudas con la propia orientación sexual en función del grupo de edad.

\begin{tabular}{lcccccccccccc}
\hline & \multicolumn{1}{c}{$18-25$} & \multicolumn{2}{c}{$26-35$} & \multicolumn{2}{c}{$36-45$} & \multicolumn{2}{c}{$46-55$} & \multicolumn{3}{c}{$56-65$} & \multicolumn{3}{c}{ Más de 65} \\
\cline { 2 - 13 } & $n$ & $\%$ & $n$ & $\%$ & $n$ & $\%$ & $n$ & $\%$ & $n$ & $\%$ & $n$ & $\%$ \\
\hline Nada & 294 & 18.5 & 504 & 48.6 & 1215 & 59.4 & 733 & 73.3 & 368 & 72.2 & 81 & 90 \\
Algo & 718 & 45.1 & 386 & 37.2 & 658 & 32.2 & 221 & 22.1 & 114 & 22.4 & 8 & 8.9 \\
Bastante & 368 & 23.1 & 116 & 11.2 & 135 & 6.6 & 39 & 3.9 & 26 & 5.1 & 1 & 1.1 \\
Mucho & 212 & 13.3 & 32 & 3.1 & 36 & 1.8 & 7 & 0.7 & 2 & 0.4 & 0 & 0 \\
\hline
\end{tabular}

Por último, un $24.7 \%$ de la muestra general afirma haber experimentado cambios en su propia orientación sexual, siendo en el grupo más joven donde se observan mayores porcentajes (véase Figura 1). De igual modo que con las dudas, existe una tendencia general por la que estos cambios se van reduciendo a medida que la edad de los grupos va aumentando, siendo estas diferencias estadísticamente significativas $\left(\chi^{2}=\right.$ 701.27; $5 \mathrm{gl} ; \mathrm{p}<.001)$.

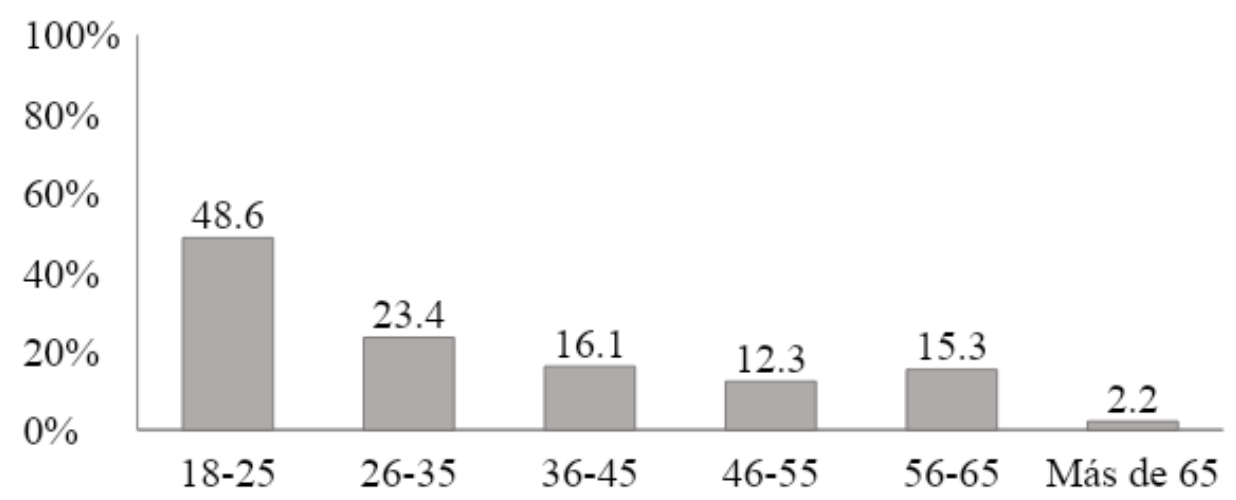

Figura 1. Porcentaje de gente que ha experimentado cambios en la orientación sexual en función de los grupos de edad.

\section{CONCLUSIONES}

Este trabajo pretendía analizar la diversidad sexual que existe en España en función del ciclo vital. Para ello, se analizaron las diferencias de los distintos grupos etarios en la orientación sexual categórica, la atracción sexual según el continuo de Kinsey, las dudas con la propia orientación sexual y los cambios en la orientación sexual.

Al igual que en otras investigaciones, los grupos más jóvenes mostraron mayor diversidad sexual, tanto en la categorización de la orientación sexual (Copen et al., 2016; Geary et al., 2018; MacCarthy et al., 2020) como en el continuo de la atracción sexual (Copen et al., 2016), utilizando más las etiquetas menos tradicionales y 
una atracción menos exclusiva hacia el otro sexo. En un estudio inglés (MacCarthy et al., 2020) que analizó las diferencias generacionales en la categorización de la orientación sexual entre una muestra de 2009 y otra de 2017, se observó que los grupos jóvenes de 2009 se identificaban más con la heterosexualidad en comparación con sus pares de 2017. Estos resultados podrían significar que los jóvenes actuales siguen menos la heteronormatividad, probablemente debido a una educación sexual más amplia y diversa (Meyer, 2010), que ayudaría a que las personas no asumieran de forma sistemática la heterosexualidad que les presupone la sociedad y se plantearan otras orientaciones que podrían estar más ajustadas a su realidad. Del mismo modo, la sociedad actual es mucho más tolerante con la homosexualidad de lo que era antiguamente (Twenge et al., 2016), lo que podría explicar que las generaciones más adultas muestren unas identidades más tradicionales, ya que habrían formado su orientación sexual en una etapa más represiva.

No obstante, esta mayor diversidad en los jóvenes también podría ser debida a una identidad menos definida, lo que también explicaría que los grupos más jóvenes presenten más dudas y más cambios en su orientación sexual que los grupos más adultos, tal y como se ha observado en la presente investigación. Recordemos que durante la adolescencia y la juventud es cuando se suele formar y consolidar la orientación sexual (Floyd y Stein, 2002; Patterson, 1995) y cuando se producen los primeros cambios en la atracción sexual (Katz-Wise, 2015), por lo que la mayor diversidad observada en los grupos jóvenes podría ser debido a que están en una etapa de exploración y cuestionamiento de su sexualidad. Sin embargo, a medida que vayan madurando y consolidando su identidad, mostrarán menos dudas, experimentaran menos cambios y, según lo que nos indican los datos, mostrarán menor diversidad.

Si bien esta investigación aporta información relevante sobre los cambios generacionales en la vivencia de la diversidad sexual, no hay que dejar de lado las limitaciones que presenta el estudio, como la utilización de autoinformes o de medidas retrospectivas, que pueden verse afectadas por los sesgos de memoria, sobre todo en la población más adulta.

A modo de conclusión, hay que destacar que el Proyecto SAFO aportará datos novedosos que no sólo tendrán utilidad científica al permitirnos alcanzar los objetivos descritos, sino que pueden llevar a importantes beneficios. A nivel social, nos ayudará a planificar intervenciones que repercutan en una mejora de la calidad de vida de las minorías sexuales y amplíen la visión de la diversidad sexual.

\section{AGRADECIMIENTOS}

Este trabajo ha sido posible gracias al proyecto de investigación UJI-B2018-42 y a la ayuda PREDOC/2017/45 de la Universitat Jaume I de Castellón.

\section{REFERENCIAS}

Ballester-Arnal, R. y Gil-Llario, M. D. (2016). Orientación del deseo sexual: Crisis y decadencia de los modelos categoriales. En A. López de la Llave (Coord.), Sexología positiva: (pp. 59-66). Universidad Nacional de Educación a Distancia.

Bauermeister, J. A., Johns, M. M., Sandfort, T. G., Eisenberg, A., Grossman, A. H. y D'Augelli, A. R. (2010). Relationship trajectories and psychological well-being among sexual minority youth. Journal of Youth and Adolescence, 39(10), 1148-1163. https://doi.org/10.1007/s10964-010-9557-y 
Carroll, J. L. (2015). Sexuality now: Embracing diversity (5th edition). Cengage Learning.

Copen, C., Chandra, A. y Febo-Vazquez, I. (2016). Sexual behavior, sexual attraction, and sexual orientation among adults aged 18-44 in the United States: Data from the 2011-2013 National Survey of Family Growth. National Health Statistics Reports (No. 88). National Center for Health Statistics.

Davison, M.K., Bershadsky, B., Bieber, J., Silversmith, D., Maruish, M.E. y Kane, R.L. (1997). Development of a brief, multidimensional, selfreport instrument for treatment outcomes assessment in psychiatric settings: Preliminary findings. Assessment, 4, 259-276.

Díaz, D., Rodríguez-Carvajal, R., Blanco, A., Moreno-Jiménez, B., Gallardo, I., Valle, C. y Van Dierendonck, D. (2006). Adaptación española de las escalas de bienestar psicológico de Ryff. Psicothema, 18(3), 572-577.

Espada, J. P., Morales, A., Orgilés, M. y Ballester, R. (2012). Autoconcepto, ansiedad social y sintomatología depresiva en adolescentes españoles según su orientación sexual. Ansiedad y Estrés, 18(1), 31-41.

Floyd, F. J. y Stein, T. S. (2002). Sexual orientation identity formation among gay, lesbian, and bisexual youths: Multiple patterns of milestone experiences. Journal of Research on Adolescence, 12(2), 167-191. https://doi.org/10.1111/1532-7795.00030

Frederick, D. A. y Essayli, J. H. (2016). Male body image: The roles of sexual orientation and body mass index across five national US Studies. Psychology of Men \& Masculinity, 17(4), 336-351. https://doi.org/10.1037/men0000031

Geary, R. S., Tanton, C., Erens, B., Clifton, S., Prah, P., Wellings, K, Mitchell, K. R., Datta, J., Gravningen, K., Fuller, E., Johnson, A. M., Sonnenberg, P. y Mercer, C. H. (2018). Sexual identity, attraction and behaviour in Britain: The implications of using different dimensions of sexual orientation to estimate the size of sexual minority populations and inform public health interventions. Plos One, 13(1), e0189607. https://doi.org/10.1371/journal.pone.0189607

Herz, M. y Johansson, T. (2015). The normativity of the concept of heteronormativity. Journal of Homosexuality, 62(8), 1009-1020. https://doi.org/10.1080/00918369.2015.1021631

Katz-Wise, S. L. (2015). Sexual fluidity in young adult women and men: Associations with sexual orientation and sexual identity development. Psychology \& Sexuality, 6(2), 189-208. https://doi.org/10.1080/19419899.2013.876445

Kinsey, A., Pomeroy, W. y Martin, C. (1948). Sexual behavior in the human male. Saunders Company.

Kinsey, A., Pomeroy, W., Martin, C. y Gebhard, P. H. (1953). Sexual behavior in the human female. Indiana University Press.

Klein, F. (1978). The bisexual option: A concept of one hundred percent intimacy. Arbor House.

MacCarthy, S., Saunders, C. L. y Elliott, M. N. (2020). Increased Reporting of Sexual Minority Orientation from 2009 to 2017 in England and Implications for Measuring Sexual Minority Health Disparities. LGBT Health, 7(7), 393-400. https://doi.org/10.1089/lgbt.2019.0181

Marchueta, A. (2014). Consecuencias del bullying homofóbico retrospectivo y los factores psicosociales en el bienestar psicológico de sujetos LGB. Revista de Investigación Educativa, 32(1), 255-271. https://doi.org/10.6018/rie.32.1.168461 
Martxueta, A. y Etxeberria, J. (2014). Análisis diferencial retrospectivo de las variables de salud mental en lesbianas, gais y bisexuales (LGB) víctimas de bullying homofóbico en la escuela. Revista de Psicopatología y Psicología Clínica, 19(1), 23-35.

Meyer, E. J. (2010). Gender and sexual diversity in schools. Springer Science \& Business Media.

Morgan, E. M., Steiner, M. G. y Thompson, E. M. (2010). Processes of sexual orientation questioning among heterosexual men. Men and Masculinities, 12(4), 425-443. https://doi.org/10.1177/1097184X08322630

Nebot-Garcia, J. E., Elipe-Miravet, M., García-Barba, M., Ruiz-Palomino, E. y Ballester-Arnal, R. (Junio, 2021a). Adaptación de la Escala de Reacciones Parentales Percibidas ante la salida del armario para población LGTB+ española: datos preliminares [poster]. II Congreso Internacional de Sexualidad: Expresando la Diversidad, Castellón de la Plana, España.

Nebot-Garcia, J. E., Elipe-Miravet, M., Martínez-Gómez, N. y Ballester-Arnal, R. (Abril, 2021b). Adaptación de la Escala de Estrés de las Minorías LGTBI en población española: datos preliminares [poster]. IV Jornadas de Investigación para el Alumnado de la Facultad de Ciencias de la Salud de la Universidad Jaume I, Castellón de la Plana, España.

Nebot-Garcia, J. E., García-Barba, M., Gil-Juliá, B., Giménez-García, C. у Ballester-Arnal, R. (2018). Comportamientos homosexuales en jóvenes heterosexuales: diferencias de género. Revista Àgora de Salut, 5, 59-68. http://dx.doi.org/10.6035/AgoraSalut.2018.5.6

Outland, P. L. (2016). Developing the LGBT minority stress measure (Doctoral dissertation, Colorado State University).

Patterson, C. J. (1995). Sexual orientation and human development: An overview. Developmental Psychology, 31(1), 3-11. https://doi.org/10.1037/0012-1649.31.1.3

Reisner, S. L., Biello, K., Perry, N. S., Gamarel, K. E. y Mimiaga, M. J. (2014). A compensatory model of risk and resilience applied to adolescent sexual orientation disparities in nonsuicidal self-injury and suicide attempts. American Journal of Orthopsychiatry, 84(5), 545-546. https://doi.org/10.1037/ort0000008

Ryff, C. (1989). Beyond Ponce de Leon and life satisfaction: New directions in quest of successful aging. International Journal of Behavioral Development, 12(1), 35-55. https://doi.org/10.1177/016502548901200102

Sandín, B., Valiente, R. M., Chorot, P., Santed, M. A. y Lostao, L. (2008). SA-45: a brief form of the SCL-90. Psicothema, 20(2), 290-296.

Twenge, J. M., Sherman, R. A. y Wells, B. E. (2016). Changes in American adults' reported same-sex sexual experiences and attitudes, 1973-2014. Archives of Sexual Behavior, 45(7), 1713-1730. https://doi.org/10.1007/s10508-016-0769-4

Walch, S. E., Ngamake, S. T., Bovornusvakool, W. y Walker, S. V. (2016). Discrimination, internalized homophobia, and concealment in sexual minority physical and mental health. Psychology of Sexual Orientation and Gender Diversity, 3(1), 37-48. http://dx.doi.org/10.1037/sgd0000146

Willoughby, B. L. B., Malik, N. M. y Lindahl, K. M. (2006). Parental reactions to their sons' sexual orientation disclosures: The roles of family cohesion, adaptability, and parenting style. Psychology of Men \& Masculinity, 7(1), 14-26. https://doi.org/10.1037/1524-9220.7.1.14 